\title{
Relationship between Toe Flexion Movement and Center of Pressure Position

\author{
Y Otake ${ }^{1,2^{*}}$, A Yozu ${ }^{1,3}$, T Fukui ${ }^{4}$, M Sumitani ${ }^{5}$ and N Haga ${ }^{1}$
}

${ }^{1}$ Department of Rehabilitation Medicine, Graduate School of Medicine, The University of Tokyo, Tokyo, Japan

${ }^{2}$ Sports Management Center, Bunkyo Gakuin University, Bunkyo-ku, Tokyo, Japan

${ }^{3}$ Department of Cognitive and Locomotor Sciences in the Super-aged Society, Graduate School of Medicine, The University of Tokyo, Tokyo, Japan

${ }^{4}$ Physical Therapy Department, Faculty of Health Science Technology, Bunkyo Gakuin University, Tokyo, Japan

${ }^{5}$ Department of Anesthesiology and Pain Relief Center, The University of Tokyo Hospital, Tokyo, Japan

*Corresponding author: Yuko Otake, Department of Rehabilitation Medicine, Graduate School of Medicine, The University of Tokyo, 7-3-1 Hongo, Sports Management Center, Bunkyo Gakuin University, 1-19-1 Mukogaoka, Bunkyo-ku, Tokyo, 113-8665, Japan, Tel: +81-3-5800-8795, Fax: +81-3-5684-2094

\begin{abstract}
In humans, the toes are important for stability while standing. We aim to elucidate the role of the toes in posture control by examining the relationship between the action of the flexor muscles and the center of pressure (COP) positions in the standing posture.

The subjects were 16 healthy young adults who were free of musculoskeletal and neurological disorders. We measured the toe plantarflexion movements, COP positions, and floor reaction force components in the standing and forward loading postures.

In subjects with more anteriorly-located COP positions during the forward body shift, the toe flexion angle was greater. Moreover, as the COP position in the static standing posture was more anteriorly located, the subjects were more likely to flex their toes with the interphalangeal joint extended.

Our findings suggest that the ability to flex the toes with extended interphalangeal joints affects stability in the static standing position. In addition, the toe flexion angles and force of pushing against the floor affect dynamic balance.
\end{abstract}

\section{Keywords}

Toe movement, Cop, Standing, Balance

\section{Introduction}

The foot is the only part of the body in contact with the ground while standing and walking, and it performs various functions. The toes, which are the only parts of the foot that are able to move, are important for stability while standing and moving [1]. While standing, the toes are in contact with the ground, the center of pressure (COP) shifts anteriorly, and the balance-controllable range increases. Consequently, the physical stability of the body is achieved. While walking, the toes stabilize the body during the entire stance phase and transmit the force during the terminal stance phase, thereby enabling propulsion [2]. In people with greater toe flexor muscle strength, the anterior limit of the COP is reportedly located at the forefoot when they lean forward from the static standing position [3]. Because of this, toe flexion is assumed to be strongly associated with COP position and anteroposterior stability.

The dysfunction of the toes is considered to cause instability in the physical balance of the body. There are reports that patients who fall have lower muscle strength of the hallux and are more likely to have toe deformities than non-fallers [4]. Therefore, decreased toe muscle strength and presence of toe deformities reduce the efficiency in shifting body weight and can increase the risk of falls [5-7]. The toe flexor muscle strength is often used as an index for reflecting toe functions. The toe muscle strength for shifting body weight forward is greater in younger people and decreases with age [3]. Other studies have used the toe muscle strength as 
an index; however, the methods used to measure and assess the toe flexor muscle strength vary among those reports [3,5,8-10]; and neither have these clarified the individual actions of the muscles nor the kinematics. Only a few electromyographic studies on gait and movement have focused on the muscle activities and actions involved in toe movement [11]. Unlike studies on the fingers [12], which have anatomical structures similar to those of the toes, no previous studies have examined each joint of the toes or their kinesiology.

Thus, in this study, with the aim of elucidating the role of the toes in posture control, we examined the relationship between the action of the toe flexion and COP positions involved in the movement of the body. Using a three-dimensional motion capture system and force plates, we measured the COP positions, floor reaction force components, and toe flexion movements corresponding to the static standing position and forward shifting of body weight. We also examined the associations between these measurements.

\section{Methods}

The participants were 16 healthy young adults ( 8 male and 8 female) who were free of musculoskeletal and neurological disorders. Demographic data are shown in Table 1. The participants were given a sufficient explanation of the study objective and methods before receiving their consent after which the measurements were obtained. This study was approved by the ethics committee of the Graduate School of Medicine at the University of Tokyo (approval no. 2889).

All the measurements were performed on the participants' right feet using the three-dimensional motion capture system, Vicon (Vicon Motion Systems, Oxford, UK) and force plates (AMTI, Watertown, MA, USA and Kistler AG, Winterthur, Switzerland). Infrared reflective markers of diameter $6.35 \mathrm{~mm}$ were applied to the dorsal aspects of the first metatarsal head, second metatarsal head, second metatarsal base, second interphalangeal (IP) joint, second toe tip (TT), and calcaneus. The error in the calculation of the threedimensional coordinates for each marker was less than $2 \mathrm{~mm}$. Before the experiments, the foot length (sagittal distance between the TT and heel markers), thickness of the second metatarsal head, and toe length (distance from the second metatarsal head to the tip) were

Table 1: Demographic variables.

No significant difference was observed in age between groups male and female, but the height and weight in male were significantly higher than that in female.

\begin{tabular}{|l|l|l|l|}
\hline \multicolumn{1}{|c|}{} & Male & Female & Over all \\
\hline No. of participants $(\mathrm{n})$ & 8 & 8 & 16 \\
\hline Age (year) & $28.2 \pm 5.8$ & $32.5 \pm 4.9$ & $30.4 \pm 5.7$ \\
\hline Height $^{*}(\mathrm{~cm})$ & $171.1 \pm 4.3$ & $161.2 \pm 5.9$ & $166.2 \pm 7.2$ \\
\hline Mass $^{*}(\mathrm{~kg})$ & $63.9 \pm 6.3$ & $49.5 \pm 5.1$ & $56.7 \pm 9.3$ \\
\hline
\end{tabular}

*significantly different between gender $(p<0.05)$. measured. The Vicon Nexus measurement software (Vicon Motion Systems) was used to calculate the three-dimensional coordinates of each marker and the dynamic data during forward shifting and toe flexion movements. Raw kinetic data were smoothed using a fourth-order Butterworth digital filter with a cutoff frequency of $16 \mathrm{~Hz}$.

The participants stood on the force plates with both feet separated by their pelvis width. To measure the reaction force of only the forefoot, the midpoint of the foot was aligned with the border of the front and back force plates with each foot placed on two force plates. The loads exerted by the anterior and posterior parts of the foot were measured separately. For the forward shifting movement, oral instructions were given for the task of voluntary maximal forward shifting of body weight without stepping forward or lifting the heels. The specified movement involved shifting the body weight forward for two beats of the metronome, which was set at 60 beats/min, and then returning to the original position for two beats. The examiner confirmed no lifting of the heels visually and from the floor reaction force data. Based on the COP coordinates of the foot, the COP positions were calculated as a percentage of the foot length measured from the heel. The static COP was defined as the mean value for $15 \mathrm{~s}$ of static standing, and the max COP was defined as the mean value of the most anteriorly-located COP position. Moreover, the floor reaction force applied to the forefoot during the forward shifting of body weight was measured. The maximum values of the anteroposterior and vertical components of the floor reaction force were averaged for three trials. The floor reaction force data were normalized by dividing the obtained values by the participants' body weights.

The participants performed toe flexion movement in the long sitting position on a bed. To examine toe flexion using the intrinsic muscles, especially the dorsal/plantar interosseous and lumbrical muscles, the assigned task was to flex the metatarsopharangeal (MP) joints with the IP joints as straight as possible. This movement was repeated three times.

While the ankle joint remained in the maximum plantar-flexed position, the movement started with the toes initially in a relaxed state. The data were normalized by dividing the obtained value by the respective times. The Vicon Bodybuilder analysis software was used to determine the center of the second MP joint. First, the center of rotation of the second MP joint in the sagittal plane (real MP Joint, RMP) was determined. The RMP was defined as the point located at $38 \%$ of the MP joint thickness from the marker on the second metatarsal head (the distance from the marker was calculated using the center of the MP joint from sagittal magnetic resonance images of the second toes of nine patients with no second toe abnormalities.) Next, the 

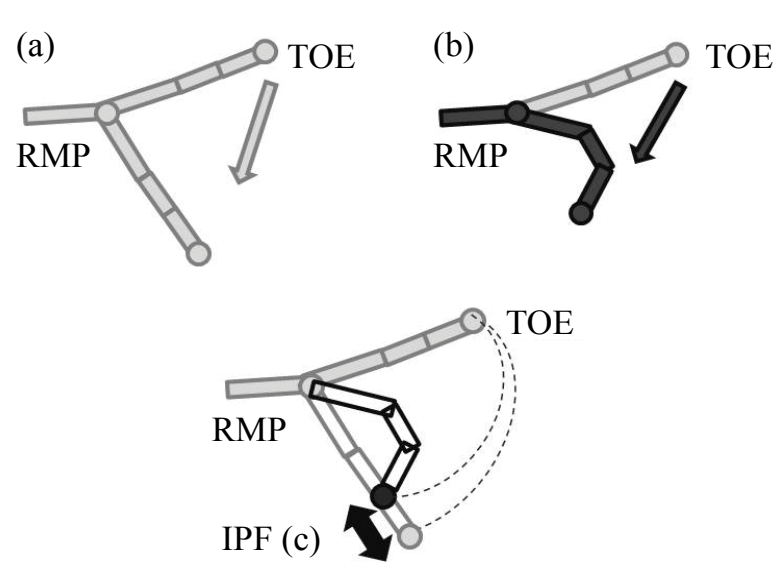

Figure 1: Definition of the IPF value.

These diagrams present the metatarsal and phalangeal bones of the foot. Diagram (a) shows the ideal trajectory of the second toe tip (TT) marker, which is a circle with the distance between the real metatarsophalangeal joint (RMP) and the TT at the start of toe flexion as a radius. Diagram; (b) shows the actual trajectory of the TT marker. The difference in the RMP-TT distance between diagrams (a) and (b) are determined and normalized. The mean of the resultant values are regarded as the IPF value (c).

toe flexion movements were assessed based on the RMP and TT coordinates. These were projected onto the two-dimensional sagittal plane to determine the trajectory of the TT around the RMP. The range of the TT movement centered on the RMP was regarded as the toe flexion angle. Then the arc with a radius equal to the distance between the RMP and TT at the start of toe flexion was plotted as an ideal trajectory to determine its coordinates. It was assumed that if the MP joints could be flexed while keeping the IP joints extended, then the TT trajectory might form an arc with a constant radius. At each point, the distances between the ideal and actual trajectories were determined and normalized by the flexion angle, and the sum of the resultant values was regarded as the IP joint flexion level (the IPF index). If the IP joints were flexed during toe flexion, then the differences between the ideal and actual trajectories as well as the IPF index increased. To normalize the variability due to the toe flexion angles, the IPF indices were divided by their respective toe flexion angles (Figure 1).

The relationship between the static COP and max COP was examined using Pearson's product-moment correlation analysis. The relationships between the COP positions, floor reaction force components (anteroposterior/ vertical component), and toe flexion angles/IPF index were examined separately using Pearson's correlation coefficients. SPSS (SPSS, Inc., Chicago, IL, USA) was used to perform the statistical analyses. The level of significance was set at $p<0.05$.

\section{Results}

\section{Center of pressure positions}

The following results are shown in terms of there

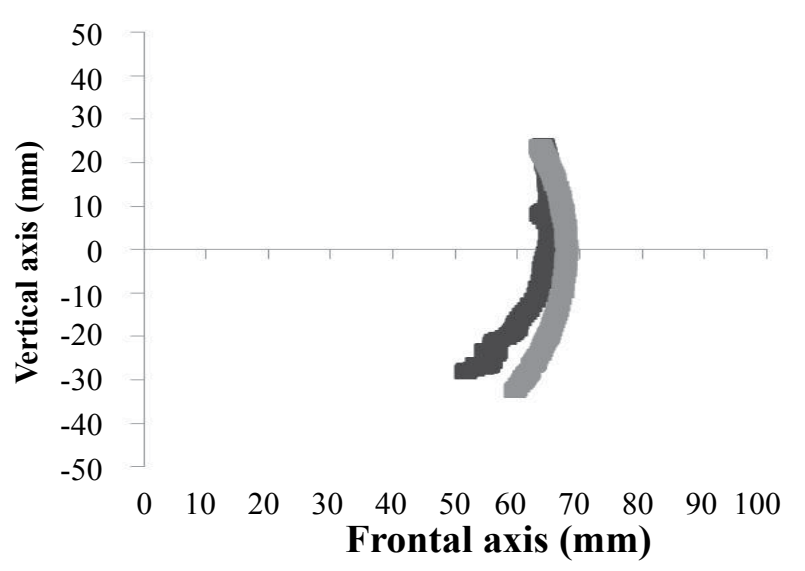

Figure 2: Trajectory of the second toe tip (TT) marker on the sagittal plane.

Both of the vertical and frontal axes indicate the distance, and the origin indicates the real metatarsophalangeal joint position. The gray line shows the ideal trajectories, and the black line shows the actual trajectories. The positive side of the vertical axis indicates that the TT marker is located at the dorsal side of the extension of the metatarsal bone, and the negative side indicates that the marker is located at the plantar side.

average (standard deviation). The static COP was located at $48.2 \%$ (SD 7.8) of the foot length from the heel. The max COP was located at $82.1 \%$ (SD 4.7). No significant correlation was observed between the static COP and max COP. For the static COP and max COP, there were no significant differences between males and females.

\section{Center of pressure position and floor reaction force components}

A significant correlation was found between the max COP and the peak of the anteroposterior component of the floor reaction force $(r=0.504, p<0.05)$. In subjects with more anteriorly-located COP positions during the forward shifting of body weight, the peak of the anteroposterior component of the floor reaction force was greater. However, there were no correlation separated by gender.

The static COP did not correlate with the peak of the anteroposterior component of the floor reaction force. Moreover, the peak of the vertical component of the floor reaction force did not correlate significantly with either the static COP or max COP.

\section{Toe flexion angles and the interphalangeal joint flexion index}

The toe flexion angle of 16 subjects was $32.8^{\circ}$ (SD $12.4^{\circ}$ ). Figure 2 shows the ideal (an arc around the RMP with radius of the RMP-TOE distance at the start of flexion) and actual trajectories of the TT obtained in one trial of toe flexion movements performed by one participant. In 13 out of 16 participants, as the toe flexion angle increased, the actual trajectory moved farther from the ideal trajectory toward the inside of the graph. In the other three participants, as the toe flexion 


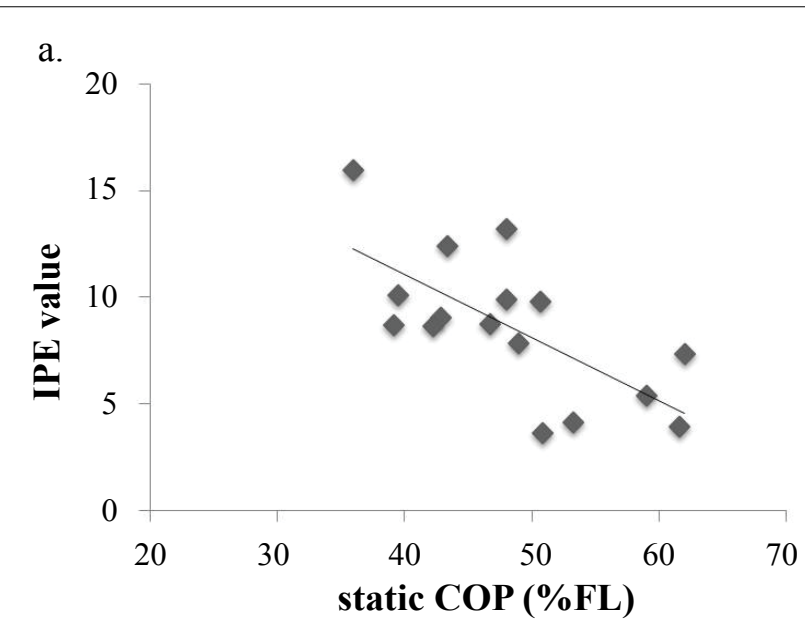

b.

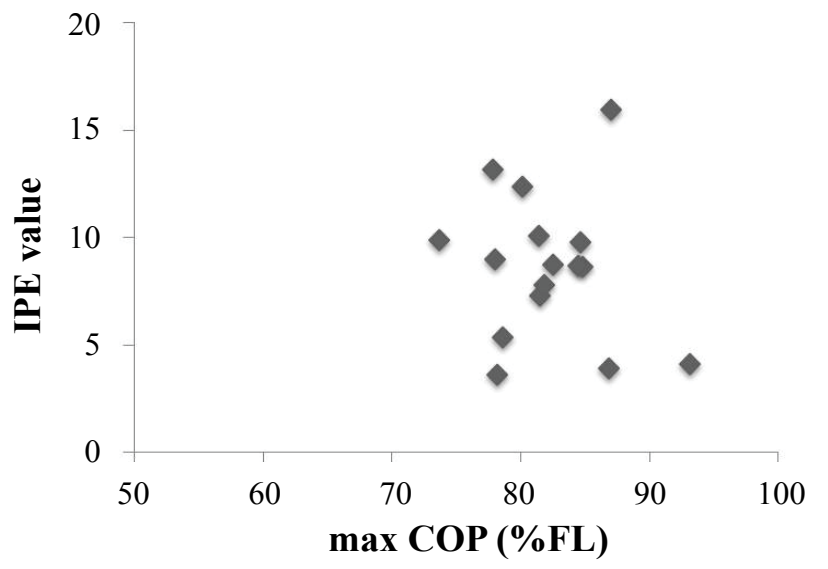

Figure 3: The relationship between the center of pressure (COP) positions and IPF values.

a) The static COP correlates with the IPF values. In subjects who were able to flex the toes with the IP joints extended, the COP in the static standing position was located in the forefoot; $b$ ) There was no correlation between the max COP and the IPF values.

angle increased, the actual trajectory crossed the ideal trajectory or moved farther from it toward the outside. The IPF index was $8.6^{\circ}$ (SD $3.4^{\circ}$ ) in 16 participants. There were no significant differences between males and females.

\section{Center of pressure positions and toe flexion angles/ interphalangeal joint flexion index}

We observed a significant correlation between the static COP and toe flexion angles $(r=0.504, p<0.05)$. As the COP position in the static standing position was more anteriorly located, the toe flexion angle was greater. Moreover, the max COP correlated strongly with the toe flexion angles $(r=0.510, p<0.05)$. In a participant with more anteriorly-located COP positions during the forward shifting of body weight, the toe flexion angle was greater. As between static COP and toe flexion angles, there was no correlation separated by gender. However, there was a significant correlation between the max COP and toe flexion angles in females $(r=0.777$, $p<0.05$ ), but not in males.
The static COP correlated significantly with the IPF index $(r=-0.559, p=0.05)$. As the static COP was more anteriorly located, the subjects were more likely to flex their toes while the IP joint remained extended. The correlation between the max COP and IPF index was not significant (Figure 3). However, there was a significant correlation between the max COP and toe flexion angles in females $(r=-0.757, p<0.05)$, but not in males.

\section{Discussion}

Previous studies on the COP position in the static standing posture showed that the COP was located at 40$50 \%$ of the foot length from the heel $[13,14]$. Shieppati, et al. reported that the anterior displacement of the COP from the static position was approximately $30-40 \%$ of the foot length. In the present study, the static and max COPs were located at $48.2 \%$ and $82.1 \%$, respectively, and the displacement was $33.9 \%$. These findings are similar to those of previous studies. However, there was no significant correlation between the static and max COP; the different mechanisms were associated with the COP positions for maintaining the static standing posture and the forward shifting of body weight.

Regarding the toe flexion movements, the distance between the RMP and TT was shorter with greater flexion angle in most participants. This indicates that the IP joints flexed as the toes flexed. Among the five muscles that control flexion, the dorsal/plantar interosseous and lumbrical muscles control the IP joint extension and MP joint flexion. If the IP joints are extended during toe flexion, then the activities of the interosseous and lumbrical muscles are expected to be larger among those responsible for toe flexion.

The static COP correlated significantly with the IPF index. In subjects who were able to flex their MP joints with their IP joints extended, the static COP was located more anteriorly in the foot. Jung, et al. compared the electromyograms of the abductor hallucis when the toes were tightly flexed or stressed as if pushing against the floor. They found that the abductor hallucis contraction was greater while pressing the toes against the floor with the IP joints extended [15]. They described the abductor hallucis activity as raising the medial arch, thereby improving balance. The abductor hallucis is attached to the base of the proximal phalanx and flexes the MP joint, and it appears to have functions similar to the intrinsic foot muscles of the other four toes (i.e., the dorsal interosseous and lumbrical muscles). Other toe flexion muscles were attached to distal phalanges. From an anatomical point of view, when the toe is flexed while the IP joints remain extended, the action of the dorsal interosseous/lumbrical muscle is expected to dominate over that of the other muscles. Moreover, the activities of these muscles seem to be the key for static balance controls. 
Regarding the relationship between the static standing position and toe functions, the measurements of quantitative factors (e.g., muscle activities and strength) indicate that the intrinsic foot muscles do not act in the static standing position [8]. In our study, the toe flexion movements in a specified manner were quantitatively analyzed, revealing an association between the static standing position and COP positions. This suggests that when the intrinsic foot muscles engage in the standing position, their functions involve foot posture or structure.

The static COP and max COP correlated significantly with toe flexion angles. In this experiment, the toe flexion angle indicated the range of TT movements centered on the RMP. In subjects with greater toe flexion angle, the static COP and max COP were more anteriorly located in the foot. It is assumed that the increase in the functional base of support with the flexing of the toes and placing them on the floor resulted in the anterior displacement of the COP. Thus, the ability to actively flex the toes and maintain the forward balance of the foot appears to be important for static balance control.

The max COP correlated significantly with the anteroposterior component of the floor reaction force. Therefore, in subjects who were able to shift their body weight to their forefeet, the forefoot force pushing back against the floor also increased. The body weight was shifted anteriorly while the toes remained on the floor, presumably increasing the backward propulsion to return to the original standing position within the specified time. This is similar to the findings of a previous study, which showed that the toe flexor muscle strength was greater in subjects who were able to shift their body weights forward [3]. Toe flexor muscle activities are considered to increase when the COP is shifted to the limit of the functional base of support, and it especially contributes to posture retention $[14,16]$. Although the toe flexor muscle strength was not measured in our study, the task of returning the anteriorly-shifted COP to the original position is similar to that in a previous study, which returned the COP to the center of the functional base of support. Thus, based on the findings of the present study, the toe flexor muscle activity can be enhanced. Therefore, the ability to flex the toes and push them against the floor may be important for dynamic balance control.

This study has some limitations. To exclude the compensation by the toe extensor and extrinsic toe flexor muscles during toe flexion and because of the difficulty in moving the toes with infrared markers attached while keeping the foot joint at a certain angle, the toe flexion movements were measured when the ankle joint remained as maximum plantar flexion. While the foot joint remained in this position, the relaxed MP joints were extended in all the subjects. When the toes were flexed with the ankle joint in plantar flexion, the stretched skin of the MP joint area may have distally shifted the marker on the second metatarsal bone. Moreover, one reason why the actual trajectory crossed the ideal trajectory or moved farther from it toward the outside may be that the toe may not have been in full extension when toe flexion began.

In conclusion, our findings revealed that in subjects who were able to flex their MP joints with their IP joints extended, the static COP was located more anteriorly in the foot. The stronger the forefoot force pushing the toe against the floor affects, the more their body weight shifted forward. In order to move their body weight forward, it is necessary to increase the flexion angles of their toe.

This study found a difference in the role of postural stability between the static COP and dynamic COP. Our findings suggested that the ability to flex the toes with the IP joints extended affects the COP position during static standing and the force of pushing against the floor affects the COP position when the body weight shifts forward during dynamic standing. This study suggests that the static COP contributes to static balance while max COP contributes to dynamic balance. Thus, choosing the right target for static COP or max COP can aid in patient treatment.

\section{Acknowledgements}

We would like to thank Dr. Hirotaka Kawano for his invaluable help with the data collection. We are also deeply grateful to Drs. Naoshi Ogata and Yasuo Nakahara for their useful suggestions. Lastly, we would like to thank Ms. Zhang Yasu for her continuing support.

\section{Conflict of Interest Disclosure}

None.

\section{References}

1. Lambrinudi C (1932) Use and abuse of toes. Postgrad Med J 8: 459-464.

2. Hughes J, Clark $P$, Klenerman $L$ (1990) The importance of the toes in walking. J Bone Joint Surg Br 72: 245-251.

3. Endo M, Ashton Miller JA, Alexander NB (2002) Effects of age and gender on toe flexor muscle strength. J Gerontol A Biol Sci Med Sci 57: 392-397.

4. Mickle KJ, Munro BJ, Lord SR, Menz HB, Steele JR (2009) ISB Clinical biomechanics award 2009: Toe weakness and deformity increase the risk of fall in older people. Clin Biomech (Bristol, Avon) 24: 787-791.

5. Mickle KJ, Munro, Lord SR, Menz HB, Steele JR (2011) Gait, balance and plantar pressures in older people with toe deformities. Gait Posture 34: 347-351.

6. Menz HB, Lord SR (2001) The contribution of foot problems to mobility impairment and falls in community-dwelling older people. J AM Geriatr Soc 49: 1651-1656.

7. Menz HB, Morris ME, Lord SR (2005) Foot and ankle characteristics associated with impaired balance and functional ability in older people. J Gerontol A Biol Sci Med Sci 60: 1546-1552. 
8. Menz HB, Zammit GV, Munteanu SE, Scott G (2006) Plantarflexion strength of the toes: Age and gender differences and evaluation of a clinical screening test. Foot Ankle Int 27: 1103-1108.

9. Senda M, Takahara $Y$, Yagata $Y$, Yamamoto $K$, Nagashima $\mathrm{H}$, et al. (1999) Measurement of the muscle power of the toes in female marathon runners using a toe dynamometer. Acta Med Okayama 53: 189-191.

10. Hamel AJ, Donahue SW, Sharkey NA (2001) Contributions of active and passive toe flexion to forefoot loading. Clin Orthop Relat Res 326-334.

11. Mann R, Inman VT (1964) Phasic activity of intrinsic muscles of the foot. J Bone Joint Surg Am 46: 469-481.

12. Srinivasan $H$ (1977) Movement patterns of intrinsic minus fingers. Role of intrinsic and extrinsic muscles in finger posture control. Ann R Coll Surg Engl 59: 33-38.
13. Murray MP, Seireg AA, Sepic SB (1975) Normal postural stability and steadiness: Quantitative assessment. J Bone Joint Surg Am 57: 510-516.

14. Schieppati M, Hugon M, Grasso M, Nardone A, Galante M (1994) The limits of equilibrium in young and elderly normal subjects and in parkinsonians. Electroencephalogr Clin Neurophysiol 93: 286-298.

15. Jung DY, Kim MH, Koh EK, Kwon OY, Cynn HS, et al. (2011) A comparison in the muscle activity of the abductor hallucis and the medial longitudinal arch angle during toe curl and short foot exercises. Phys Ther Sport 12: 30-35.

16. Kelly LA, Kuitunen S, Racinais S, Cresswell AG (2012) Recruitment of the plantar intrinsic foot muscles with increasing postural demand. Clin Biomech (Bristol, Avon) 27: 46-51. 